\title{
Design and Performance Analysis of Energy Aware Routing Protocol for Delay Sensitive Applications for Wireless Sensor Networks
}

\author{
NANDINI PRASAD K S ${ }^{1}$, PUTTAMADAPPA C ${ }^{2}$ \\ ${ }^{1}$ Dr. Ambedkar Institute of Technology, Department of ISE, Bangalore \\ ${ }^{2}$ Sapthagiri College of Engineering, Department of ECE, Bangalore \\ E-mail:1 ${ }^{2}$ Inandiniks1@gmail.com,2puttamadappa@gmail.com
}

\begin{abstract}
Wireless Sensor Networks (WSNs) are emerging network technology with innumerable applications. But security and energy constraints reduce its successful deployments. The nodes in network are greatly involved in transmissions and other processing operations for maintenance other than establishing or handling a call. Due to limited processing ability, storage capacity and most importantly the available battery power of the nodes, it is required to minimize the transmission power and the amount of data transmitted, for efficient operation. This paper presents a power aware routing protocol designed for wireless sensor networks. The proposed routing protocol is an extended and enhanced version of Dynamic Source Routing protocol. It adds energy awareness to the existing implementation of DSR protocol. Energy metric is considered during route selection process to choose an optimal path in terms of overall energy of the nodes along the path, and "low energy notification" method is used during route maintenance process to increase the lifetime of the 'bridge' nodes to avoid network partitioning. The performance of DSR protocol and Energy Aware DSR (EADSR) protocol are compared through NS2 simulation under different scenarios. In all the cases, it is seen that EADSR protocol out-performs DSR protocol by energy saving in efficient manner.
\end{abstract}

Keywords: Transmission, DSR, Energy aware, routing protocol, WSNs.

\section{INTRODUCTION}

The deployment of specialized category of sensor networks is increasing drastically to monitor the physical environments in variety of delay sensitive applications such as military applications, agriculture, medical transport, industry etc. The most important application of wireless sensor network is to monitor the critical condition where end to end delay becomes bottleneck [1]. The monitoring applications involve the sensing of information during emergency state from the physical environment where the network of sensor is deployed. During critical conditions like explosions, fire and leaking of toxic gases, there is a need of system which should be fast enough and must respond within a fraction of seconds. These leads to a big challenge for researchers to develop a fast, reliable and fault tolerant channeled sensor networks during emergency and critical conditions to base station that receives the events [2].

Wireless Sensor Network (WSN) [3] has been regarded as special category of Ad hoc Network that may be used for a specific targeted application. Wireless Sensor Networks mainly consists of thousands of low cost, small size and battery powered sensor nodes which has more potentials than others Ad hoc networks to be deployed in many emerging areas. There is a need of designing a robust, energy efficient, minimum delay routing protocols to route the packets in these kinds of networks [4]. Out of so many routing protocols Dynamic Source Routing protocol realized to be promising one. A significant design challenge is presented by Routing to meet the various design challenges to cater the real time application requirements in wireless sensor networks (WSNs) communication. In this paper, we design and 
develop a modified Dynamic Source Routing protocol, termed as Energy Aware DSR (EADSR) which aims to minimize delay on multipath routing, end to end delay and consumes very less energy to provide enhance quality of Service for delay applications in Wireless Sensor Networks. We have considered main effecting factors such as end to end delay and unreliable of wireless channel links to routes derive a routing metric used by the routing protocol to determine the cost linked with separate routes. The route with minimum end to end delay for transmissions is selected. Although this scheme can somewhat increase the latency of the data transfer but it results in a significant power saving and long lasting routes. It maintains the existing performance of DSR and adds to it the power awareness to increase the network lifetime. The most significant benefit by adding energy awareness is that it avoids, for a long time, partitioning of network into non-communicating disjoint sets.

The paper aims at discovering an efficient power aware routing scheme for ad hoc network with less or no mobility. Simulation result shows that the proposed scheme EAR outperforms other existing routing protocols in terms of different energy related parameters. At the time route selection, EAR take crucial thing like battery status of the path into consideration while also giving importance to delay. With this main factor in consideration, EAR always select less congested and more stable route for data delivery. In this paper, an attempt has been made to evaluate the performance of DSR routing protocol using some simulation network models, to investigate how well this protocol performs on WSNs using NS-2 simulator. The performance study will focus on the impact of the network size, network density (up to 500 nodes), and the number of sources (data connections). The performance metrics used in this work are average end-to-end delay, packet delivery fraction and average energy consumption per delivered packet. Simulation results reveal better performance for the modified DSR protocol, most importantly when used for routing in unreliable wireless channel link conditions with high packet error rates. The remaining sections are organized as follows: In section 2, the related work is discussed. Section 3 explains the features of DSR protocol. Section 4 covers the implementation part followed for DSR and EADSR. Section 5 deals with the simulation carried out in the implementation. Section 6 describes the results and performance analysis of DSR and EADSR. Last section 7 , deals the conclusions and future work to be carried out.

\section{RELATED WORK}

Power aware routing (PAR) protocols have been proposed in response to the energy conservation requirement at the network layer. The energy here refers to the energy utilized by the nodes to transmit and receive packets, i.e. the energy utilized by the communication subsystem. The energy required for data processing and other auxiliary processing is not considered here because the processing energy depends on the mission of each node and is usually negligible when compared to the power required by the communication interface. An early goal of PAR was to select the best path such that the total energy consumed by the network is minimized. The basic approach is to minimize the average energy consumed per packet (as it traverses the network) or per unit flow. As in the case of minimum-hop routing, one serious drawback of this approach is that nodes will have a wide difference in energy consumption. Nodes on the minimum energy paths will quickly drain out while the other nodes remain intact. This results in an early death of some nodes.

In scenarios where the nodes need to work collaboratively, another objective of PAR is proposed: maximize the time taken by the first node/sensor to fail because it runs out of battery power. This time is known as the system lifetime. Coyle et al. [5] proposed a set of power-aware metrics based on battery power consumption at nodes. These metrics can be easily incorporated into existing routing protocols. One of the metrics, the minimum cost/packet metric, aims to maximize the lifetime of all nodes in the network. The minimum-cost routing algorithms using this metric achieved significant reduction in cost/packet over minimum-hop routing. Chen et al. [6] proposed an energy-conserving routing protocol to maximize the system lifetime by balancing the energy consumption among the nodes in proportion to their energy reserves. Norouzi et al. [7] investigated the minimum energy and maximum lifetime issues together and revealed that the two goals are not compatible. As a trade-off, he proposed a conditional max-min battery capacity routing scheme which chooses the shortest path if all nodes in all possible routes have sufficient battery capacity. When the battery capacity of some nodes fall below a predefined threshold, routes going through these nodes will be avoided, and therefore the time until the first node power-down is extended. These proposed schemes embed the energy awareness (each node is aware of its existing battery reserves) into the routing protocol and were proposed for a sensor network, where all the nodes are treated identical in terms of available 
resources and functioning roles. In addition, those schemes are more suitable for static networks because the benefits come from the even distribution of traffic among different nodes. While the nodes are moving independently, the savings provided by these algorithms, if any, is negligible because of the difficulty of real-time reconfiguration.

\section{FEATURES OF DYNAMIC SOURCE ROUTING PROTOCOL}

Dynamic source routing protocol(DSR) is an ondemand routing protocol designed to restrict the bandwidth consumed by control packets in ad hoc wireless networks by eliminating the periodic tableupdate messages required in the table-driven approach [8]. The major difference between this and other on-demand routing protocols is that it is beacon-less and hence does not require periodic hello packet (beacon) transmissions, which are used by a node to inform its neighbors of its presence. The basic approach of this protocol (and all other on-demand routing protocols) during the route construction phase is to establish a route by flooding Route Request packets in the network. The destination node, on receiving a Route Request packet, responds by sending a Route Reply packet back to the source, which carries the route traversed by the Route Request packet received. Consider a source node that does not have a route to the destination. When it has data packets to be sent to that destination, it initiates a Route Request packet. This Route Request is flooded throughout the network. Each node, upon receiving a Route Request packet, rebroadcasts the packet to its neighbors if it has not forwarded it already, provided that the node is not the destination node and that the packet's time to live (TTL) counter has not been exceeded. Each Route Request carries a sequence number generated by the source node and the path it has traversed. A node, upon receiving a Route Request packet, checks the sequence number on the packet before forwarding it. The packet is forwarded only if it is not a duplicate Route Request. The sequence number on the packet is used to prevent loop formations and to avoid multiple transmissions of the same Route Request by an intermediate node that receives it through multiple paths. Thus, all nodes except the destination forward a Route Request packet during the route construction phase. A destination node, after receiving the first Route Request packet, replies to the source node through the reverse path the Route Request packet had traversed. Nodes can also learn about the neighboring routes traversed by data packets if operated in the promiscuous mode (the mode of operation in which a node can receive the packets that are neither broadcast nor addressed to itself). This route cache is also used during the route construction phase. The important features include: Routes maintained only between nodes who need to communicate, reduces overhead of route maintenance, Route caching can further reduce route discovery overhead, a single route discovery may yield many routes to the destination, due to intermediate nodes replying from local caches and its limitations are: Packet header size grows with route length due to source routing, Flood of route requests may potentially reach all nodes in the network, care must be taken to avoid collisions between route requests propagated by neighboring nodes.

\section{DESIGN OF EADSR}

The DSR protocol is composed of two mechanisms that work together to allow the discovery and maintenance of source routes in the ad hoc network. They are route discovery and route maintenance mechanisms. Modifications are proposed for each mechanism to add power awareness to DSR and help in efficient usage of energy in the nodes [9]. Before discussing the changes in design, some assumptions are made to simplify the design process. It is assumed that all nodes wishing to communicate with other nodes within the sensor network are willing to participate fully in the protocols of the network. In particular, each node participating in the network should also be willing to forward packets for other nodes in the network. Error in transmission medium is assumed to be zero. Nodes within the sensor network that the network is static and wireless transmission range of the particular underlying network.

There have been two major modifications done to DSR algorithm: Change the routing algorithm DSR so that, given two nodes between which we must establish a multi-hop path. The path chosen among all the possible ones is such that passing through the nodes along the path have a higher level of energy and less delay at that moment.

Modify the algorithm so that, when the energy of a node which is forwarding data within a multi-hop path reaches a level below or equal to a certain threshold of the initial energy. This node will request the neighbors to look for another path, if available. Thus it avoids consuming the residual energy in a short time and also network partitions. 


\subsection{Modifications to route discovery phase}

When a node receives a Route Request (RREQ) which is not a final receipt, before forwarding RREQ packets to neighboring nodes, it waits for a pseudo random amount of time interval. This time interval is estimated from a uniform distribution of probability between 0 and a steady BroadcastJitter. The standard value of BroadcastJitter is 0.01 seconds.

The basic idea of the EADSR is that such a delay, instead of being random, should be inversely proportional to the level of residual energy of the node in that moment. In this way, the first RREQ that will come to destination node will be channeled through the best route from the point of view of "overall" energy. It is nothing but the sum of the energy levels of the intermediate nodes is maximum compared to all other possible paths from source to destination. Consequently, the total delay between the sending of RREQ by the source and the receipt by destination will be minimum.

\subsection{Modifications to route maintenance phase}

In the traditional DSR algorithm, once a certain path is chosen for sending a stream of packets to a certain destination, it tends to be used until 'one or more' nodes that compose it are no longer available (consume all their energy, moving outside of the range of neighboring nodes). The consequence is that the nodes which consume more energy in transmission and reception tend to drain rapidly than. This phenomenon may not be desirable, as some of these nodes may subsequently have the data to be transmitted and in the absence of energy, it would not be able to do so. Even in the absence of such a requirement, it may still be desirable to maintain a balance in the energy consumption of nodes, and the consequent breaks of the links, which sooner or later leads to the division of the network into two or more non-communicating partitions.

To avoid the above consequence, the following changes are incorporated to DSR algorithm:

1. When the energy of an intermediate node $X$, forwarding data within a multi-hop path reaches a level less than or equal to a certain threshold of the initial energy. The node X sends a special broadcast packet to its neighbors $\mathrm{Y}$, containing a flag in the header "low energy" set to 1, with which it implies asks not to continue to forward packets to it, if there are other paths to the destination node.
2. Each neighbor $Y$ which receives this broadcast packet of "low energy" or "low energy notification", it eliminates the paths containing the link $\mathrm{Y}->\mathrm{X}$ from its Route Cache , which is considered to be "virtually" dead (although in fact it is still working, not having $\mathrm{X}$ completely exhausted of its energy). If there are packets that have yet to be forwarded along the link Y $\rightarrow>$ X, they are still sent, to avoid destabilizing the network.

3. If one of the neighbors $\mathrm{Y}$ receives a packet to be forwarded, containing in its path the link Y-> X, Y will attempt to save (salvage) the pack et, or will generate a Route Error to be sent in broadcast, so that it reaches the source of this packet, which will attempt to re-send the package using a different path, not containing the link $\mathrm{Y}->\mathrm{X}$.

4. If the source cannot find other paths in its Route Cache, and on sending of a Route Request, if it appears that the node $\mathrm{X}$ is still active, and it appears to be the only "bridge" node to a certain destination, it will be still used to forward packets.

\section{SIMULATION ENVIRONMENT}

The simulation environment is created with an network simulator NS2 [17] in the area of $1000 \mathrm{~m} \mathrm{x}$ $1000 \mathrm{~m}$. Simulation is carried out for the scenarios containing 10, 20 and 30 nodes with the simulation time of 200 seconds and each node having the initial energy of 100 joules.

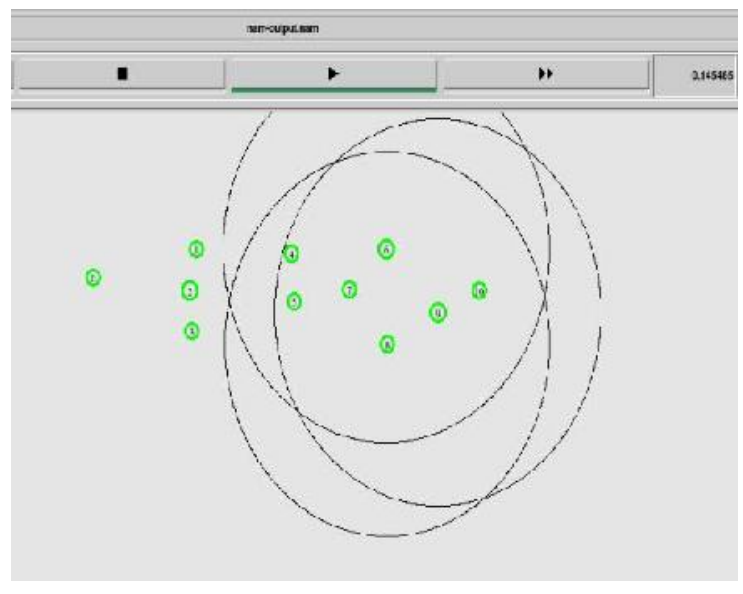

Fig. 1. Simulation scenario with all the nodes having equal energy 
Simulation is done by considering the channel as wireless, propagation as two-ray ground, queue size at each node is set to 50 where the queue can hold maximum of 50 packets at each node, antenna type is omnidirectional where it can receive and transmit in all directions.

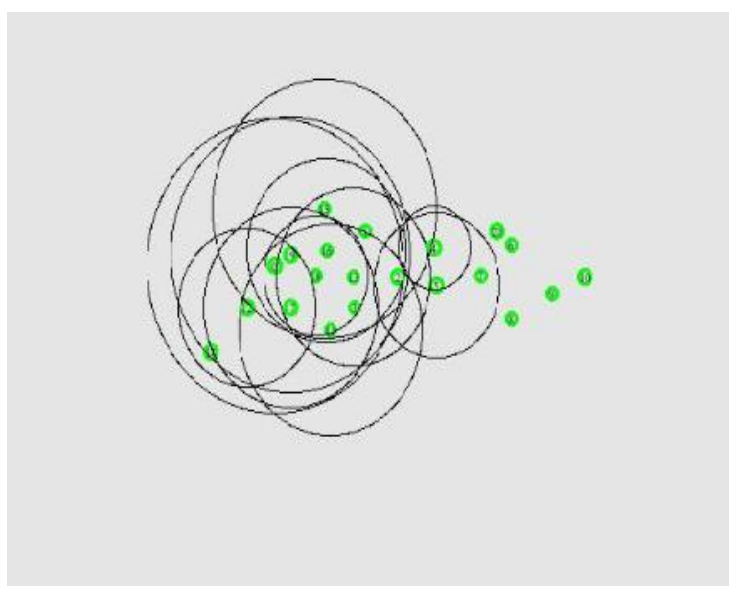

Fig. 2. Route request flooding

The constant bit rate (CBR) model [9] was used for transmission of data. In this model the data packet is transmitted periodically with a packet size of 512 bytes from source to destination.

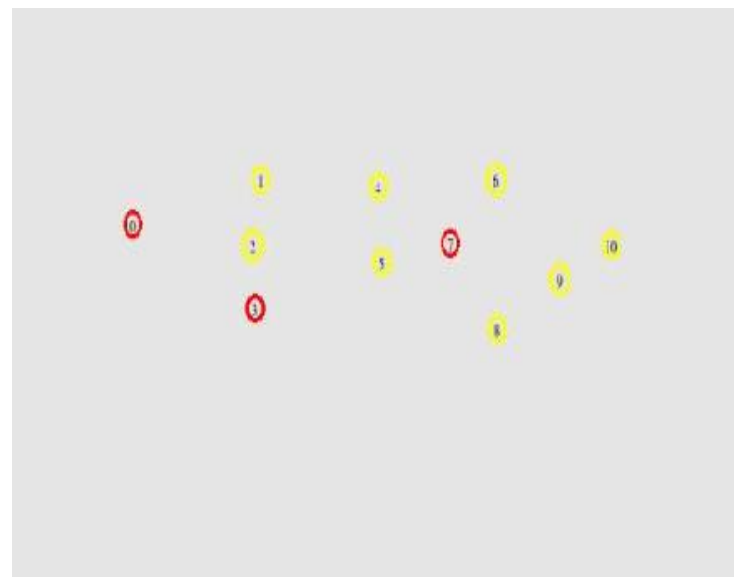

Fig. 3. Red nodes with energy below threshold

Analysis of these packet scan determine the performance effects from parameter variation, routing protocols and are performed using awk scripts. The nam file contains information on the topology such as node movement traces and events.

\section{RESULTS AND PERFORMANCE ANALYSIS}

The simulation is carried out in NS2 simulation environment for DSR and EADSR protocols to obtain certain parameters for comparison. Trace files and NAM files are generated for each simulation. Trace files contain various values like packets sent, packets received, energy of nodes, and so on. These values represent the network behavior for the particular topology. The required values are extracted from Trace files using AWK scripts. Graphs are plotted for the extracted values like Exhaustion time of first node, Network Lifetime, Packet Delivery Ratio, and Residual Energy at the end of the simulation.

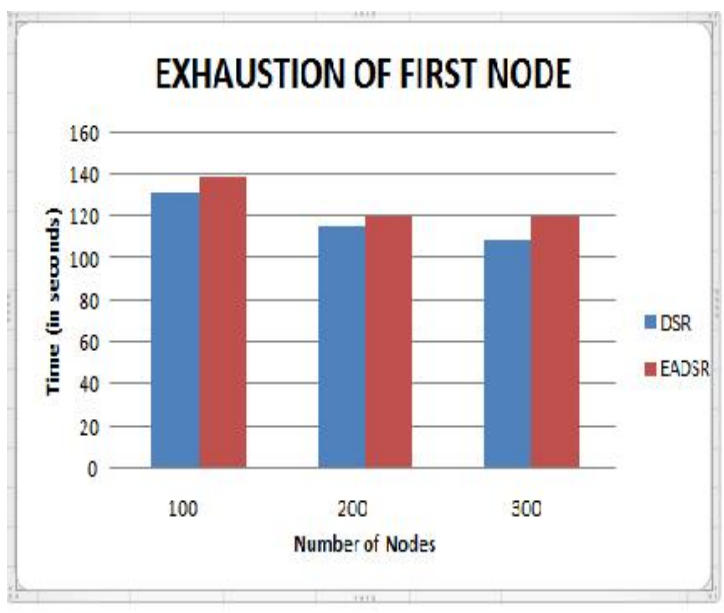

Fig. 4. Comparison of exhaustion of first node, EADSR outperforms.

When a node dies, the other nodes that can be reached only through that node become unreachable. This is because network starts to destabilize with the energy exhaustion of first node. The figure 4 shows the exhaustion time of first node when using DSR is low compared to EADSR. This clearly indicates that EADSR maintains the network stability for a long time compared to DSR. 


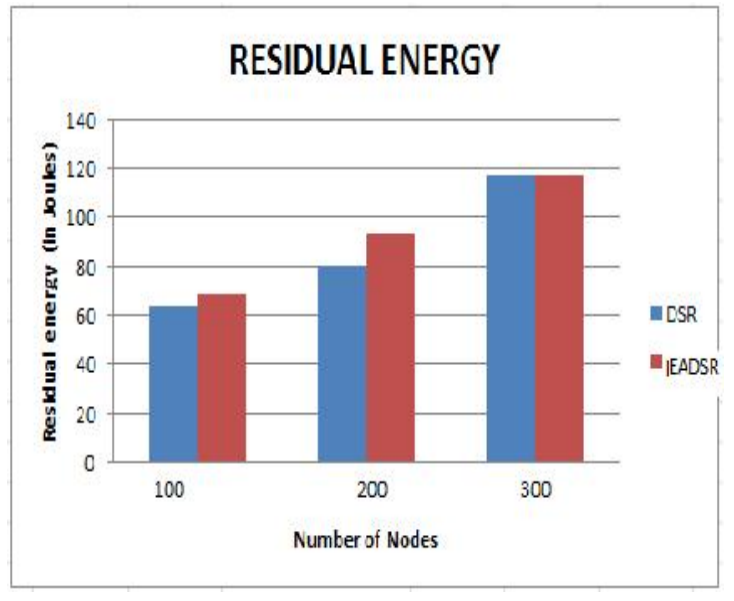

Fig. 5. Residual Energy versus Number of sensor nodes

The total residual energy gives the total energy consumed in the network before the end of the simulation. This metric doesn't give much information about how efficiently the energy is used but provides information about how much energy is saved during the routing process. The figure 5 shows that both DSR and EADSR perform equally well in terms of residual energy.

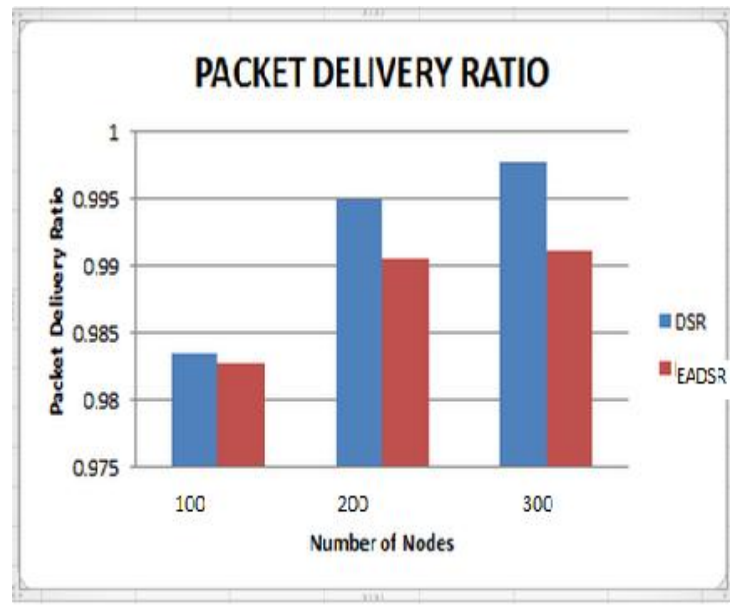

Fig. 6. Packet Delivery Ratio versus Number of sensor nodes

The ratio of number of data packets sent by source through the network and data packets received at the destination gives the packet delivery ratio. This ratio provides the information about how efficiently the routing is done and how successful the network is in data transmission. EADSR performs equally well as DSR but that value is negligible when compared with the energy efficiency gained.

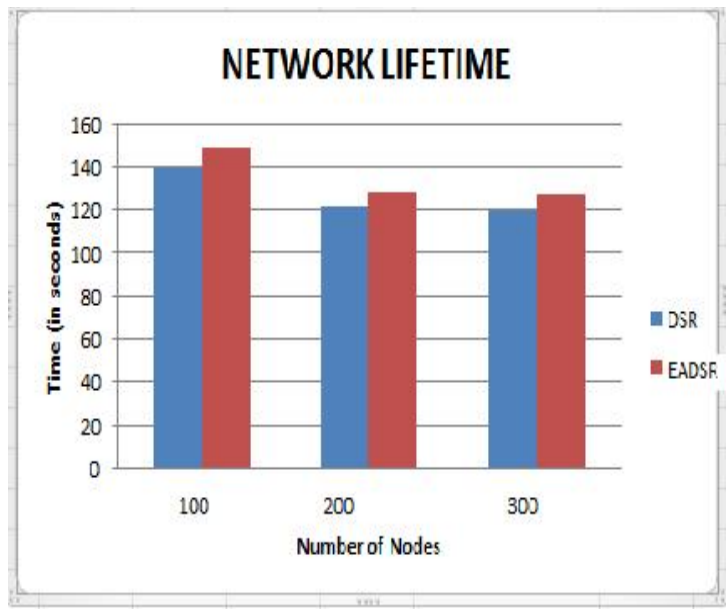

Fig. 7. Time versus Number of sensor nodes

The time taken for $30 \%$ of the nodes in the network to completely lose their energy is taken as the Network lifetime. As soon as more nodes start to lose their energy, the network become unstable and paves way for Route Request Flooding in the network. Many of the nodes become unreachable due to death of 'bridge' nodes. Hence Network lifetime is one of the most important metrics to measure the network performance. The figure 7 reveals that EADSR, compared to DSR, maintains the network stability for a long time, and avoids the partition of network into non-communicating disjoint sets. The network will be available for data transmission for a long time compared to DSR. Thus EADSR achieves the goal of efficiently using the energy.

\section{CONCLUSION AND FUTURE WORK}

Efficient usage of energy is one of the most important concerns in sensor network, especially while designing a routing protocol. This paper aims at discovering an efficient power aware routing scheme for ad hoc network with less or no mobility. Simulation result shows that the proposed scheme EADSR outperforms other existing routing protocols in terms of different energy related parameters. At the time route selection, EADSR take crucial thing like battery status of the path into consideration while also giving importance to delay. With this main factor in consideration, EADSR always select less congested and more stable route for data delivery. Although this scheme can somewhat increase the latency of the data transfer but it results in a significant power saving 
and long lasting routes. It maintains the existing performance of DSR and adds to it the power awareness to increase the network lifetime. The most significant benefit by adding energy awareness is that it avoids, for a long time, partitioning of network into non-communicating disjoint sets. Further the EADSR protocol may be used in real life scenario is straight forward but may require little modifications. More wide research is necessary to further increase the efficient usage of energy in sensor network.

\section{REFERENCES}

[1] I. F. Akyildiz and M. C. Vuran, "Wireless Sensor Net- works," 1st Edition, John Wiley \& Sons, Ltd, Chichester, 2010. doi:10.1002/9780470515181

[2] Á. Lédeczi, A. Nádas, P. Völgyesi, G. Balogh, B. Kusy, J. Sallai, G. Pap, S. Dóra, K. Molnár, M. Maróti and G Simon, "Countersniper System for Urban Warfare," ACM Transactions on Sensor Networks, Vol. 1, No. 2, 2005, pp. 153-177. doi:10.1145/1105688.1105689

[3] T. He, S. Krishnamurthy, J. A. Stankovic, T. Abdelzaher, L. Luo, R. Stoleru, T. Yan, L. Gu, G. Zhou, J. Hui and B. Krogh, "VigilNet: An Integrated Sensor Network System for EnergyEfficient Surveillance," ACM Transactions on Sensor Networks, Vol. 2, No. 1, 2006, pp. 138.doi:10.1145/1138127.1138128

[4] Mourtaji, I., et al. "A New Technique for Adapting SIP Protocol to Ad Hoc Networks: VNSIP (Virtual Network for SIP) Illustration and Evaluation of Performance" International Journal of Computer Networks and Communications Security 1.1 (2013).

[5] S. Bandyopadhyay and E. Coyle, "An Energy Efficient Hierarchical Clustering Algorithm for Wireless Sensor Networks," Proceedings of the 22nd Annual Joint Confer-ence of the IEEE Computer and Communications Societies(INFOCOM2003), San Francisco, 30 March-3 April 2003, pp. 1713-1723.

[6] Y. Chen and Q. Zhao, "On the Lifetime of Wireless Sen-sor Networks," IEEE Communications Letters, Vol. 9, No. 11, 2005, pp. 976-978.doi:10.1109/LCOMM.2005.11010

[7] A. Norouzi and A. Sertbas, "An Integrated Survey in Efficient Energy Management for
WSN Using Architec-ture Approach," International Journal of Advanced Net-working and Applications, Vol. 3, No. 1, 2011, pp. 968977.

[8] A. Kanavalli, D. Sserubiri, P. D. Shenoy, K. R. Venugopal and L. M. Patnaik, "A Flat Routing Protocol for Sensor Networks," Proceeding of International Conference on Methods and Models in Computer Science, Delhi, 14-15 December 2009, pp. 1-5.

[9] Yi hua zhu, Wan-deng wu, Jian pan, Yi-ping tang, An energy efficient data gathering algorithm to prolong lifet ime of wireless sensor networks"Computer Communications 33(2010) 639-647.

[10]F.Bajaber, I.awan, “Centralized dynamic clustering for wireless sensor network"in International Conference on Advanced Information Networking and Applications Workshops, 2009, pp 193-198.

[11] Ameer Ahmed Abbasi, Mohamed Younis, "A Survey on Clustering Algorith ms for Wireless Sensor Networks", Computer Communications 30 (2007) 2826-2841.

[12] Kemal Akkaya, Mohamed Younis, “A Survey on routing protocols for Wireless Sensor Networks", Ad Hoc Networks 3 (2005) 325349.

[13] Miau Yu, Jason H.Li and renato Levy, "Mobility Resistant Clustering in Multi-Hop Wireless Networks, Journal of Networks, Vol.1, No.1, May 2006.

[14] O. Younis, S. Fahmy, "HEED: A Hybrid,Energy-Efficient, Distributed clustering approach for Ad Hoc sensor networks , IEEETransactions on Mobile Computing 3 (4) (2004) 366-379.

[15] S. Lindsey, C. Raghavendra,"PEGASIS: Power Efficient Gathering in Sensor Information Systems," IEEE Aerospace Conference Proceedings, 2002, Vol. 3. No. 9-16, pp. 11251130.

[16]J. Kulik, W. R. Heinzelman, and $H$. Balakrishnan, "Negotiation-based protocols for disseminating information in wireless sensor networks," Wireless Networks, Volume: 8, pp. 169-185, 2002.

[17] NETWORK SIMULATOR NS2 : WWW.ISI.EDU/NS/NSNAM/NS 


\section{AUTHOR PROFILES:}

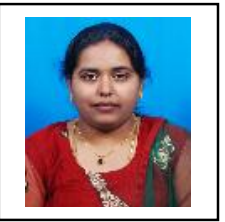

Prof. Nandini Prasad K S received degree in computer science \& engineering from PESIT, Bangalore and PG degree from VTU, Belgaum. She is a research student of

Kuvempu University. She is pursuing Ph.D under the guidance of Dr. Puttamadappa C. Currently; she is an Associate Professor at Dr. AIT, Bangalore.

Dr. C Puttamadappa obtained his B.E degree from Mysore University, PG degree from Bangalore University and Doctral degree from Jadavpur University, Kolkatta. Currently, he is working as Principal and

Professor at Sapthagiri college of Engineering, Bangalore. His research interest's areas are Devices \& Networks. 